\title{
INVESTIGACIONES
}

\section{Influencia del período práctico de formación en las creencias sobre la enseñanza, de futuros docentes}

\author{
Influence on the practical training period in beliefs \\ about teaching of future teachers
}

\author{
María Sotos Serrano ${ }^{a}$ \\ ${ }^{a}$ Departamento de Matemáticas, Universidad de Castilla-La Mancha, España. \\ maria.sotos@uclm.es
}

\section{RESUMEN}

En este trabajo se analizan los sistemas de creencias sobre la enseñanza de 6 casos de estudiantes del Grado de Maestro de la Facultad de Educación de Albacete y la influencia que el período práctico de formación pueda tener en la consolidación de dichos sistemas de creencias. Se parte de la conjetura de que estos sistemas de creencias se van consolidando a lo largo de toda la trayectoria escolar y de que la influencia de la formación universitaria es escasa. Para analizar esto se han utilizado diferentes técnicas de investigación, todas ellas desde una perspectiva cualitativa, partiendo de la tipología de tendencias didácticas de R. Porlán (1989) según la reelaboración de J. Carrillo (1998). Las conclusiones principales son que los sistemas de creencias se ajustan mejor al modelo de enseñanza espontaneísta y que el período de prácticas sólo sirve para reforzar el sistema de creencias que ya posee el alumnado.

Palabras claves: concepciones del profesor, docencia, prácticum, formación de profesores, estudio de casos.

\begin{abstract}
This paper analyzes the belief systems on the teaching of 6 cases of students of the Master's Degree of the Faculty of Education of Albacete and the influence that the practical training period may have on the consolidation of these belief systems. It is based on the guess that these belief systems are consolidated throughout the entire school career and that the influence of university education is scarce. In order to achieve this, different research techniques have been used, all from a qualitative perspective, based on the typology of didactic tendencies of R. Porlán (1989) according to the reworking of J. Carrillo (1998). The main conclusions are that belief systems are better suited to the spontaneous teaching model and that the traineeship only serves to reinforce the belief system that students already possess.
\end{abstract}

Key words: teacher conceptions, teaching, practicum, teacher education, case studies. 


\section{INTRODUCCIÓN}

El término creencias presenta una gran variedad de definiciones y comparte el mismo campo semántico que concepciones o actitudes (Vila y Callejo, 2005). Así, M. F. Pajares (1992) atribuye a las creencias los mismos componentes que aparecían en la formulación clásica de Gordon W. Allport del concepto de actitud: aspectos cognitivos, afectivos y conativos. Cognitivos en la medida en que resumen ciertos conocimientos relevantes adquiridos mediante determinadas experiencias personales, afectivos por su capacidad para desencadenar emociones o sentimientos en el sujeto, y conativos en el sentido de que ejercen una influencia directa o dinámica sobre la conducta.

También conviene recordar que los sistemas de creencias suelen incluir gran cantidad de material episódico (Abelson, 1979), o lo que es lo mismo, el conocimiento de sentido común de cualquier persona tiene un carácter fundamentalmente intersubjetivo, y estas construcciones intersubjetivas del sentido común siempre se realizan con referencia a la situación real, biográficamente determinada, de cada sujeto (Schutz, 1974).

En el ámbito de la educación, el estudio de las creencias del profesorado comienza a desarrollarse a partir de la publicación, en 1968, del libro La vida en las aulas (Jackson, 1992), con estudios descriptivos del sistema de creencias del docente en un momento dado. Pero los procesos de pensamiento del profesorado se configuran a lo largo de un proceso que incluye el antes, el durante y el después de la enseñanza (Shulman, 1989), de manera que sus experiencias biográficas, más allá de los límites de su período de formación y de práctica profesional, tienen una importancia radical a la hora de descubrir sus creencias personales, muchas veces implícitas, que se conforman a través de lo que se puede denominar conocimiento práctico o artesanal (Leinhardt, 1990). Todos los modelos que pretenden explicar la socialización docente incluyen la biografía (entendida como experiencias previas al período de formación docente en sentido estricto) como una de las variables a tener en cuenta (Rodríguez, 2002a), en una especie de proceso de socialización en su sentido más amplio, pues no se limita a los procesos específicos de formación profesional universitaria.

Esta génesis de las creencias como resultado de la socialización docente, también tiene implicaciones directas respecto a los procesos de formación del profesorado. Pese a que el modelo de formación del profesorado, en el que primero se aprende la teoría y después esta es aplicada en la práctica escolar, se ha mostrado escasamente eficaz (González y Fuentes, 1994), sigue siendo el más común en las universidades españolas. Pero el conocimiento práctico es el conocimiento que procede de la práctica, de ahí que sea desde la práctica escolar de los futuros docentes desde donde se puede construir cualquier proceso de formación que aspire a ser significativo. No puede pretenderse abordar las creencias implícitas del alumnado mediante sistemas de instrucción directa, "si los formadores de profesores tienen que provocar que los estudiantes para profesor vuelvan a pensar sobre estas creencias, se deben crear situaciones donde se enfrenten a ellas y las reconsideren" (Wilcox, Lanier, Schram \& Lappan, 1992, p. 2). En definitiva, el estudio de la formación de las creencias del profesorado tiene un componente práctico final: ¿de qué manera puede resultar más eficaz elaborar procesos de instrucción docentes para explicitar las creencias de las/os futuras/os maestras/os?, ¿cómo plantear procesos de innovación didáctica que resulten significativos en el ámbito de las creencias? Son cuestiones relevantes para el proceso de formación docente que, pese a los trabajos acumulados, siguen sin respuestas 
claras. Las investigaciones muestran la dificultad de conseguir cambios significativos en los sistemas de creencias de futuros docentes y que la enseñanza universitaria en España carece de la capacidad suficiente para influir en este terreno.

\section{FUNDAMENTACIÓN}

En 1992 se publican dos artículos en los que se realiza una revisión de los estudios sobre creencias y concepciones del profesorado. Uno referido a la enseñanza en general (Pajares, 1992) y otro a la enseñanza de las matemáticas en particular (Thompson, 1992). Pajares señala la variedad de métodos desde los que se han estudiado las creencias del profesorado, pero sugiere la conveniencia del uso de métodos cualitativos, así como la necesidad de analizar las creencias de los/as estudiantes para profesor/a. Plantea que el profesorado se encuentra encarcelado en el círculo académico, pues pasa de la vida discente a la vida docente sin cambiar de contexto, con lo que existen menos posibilidades de que sus creencias sobre la enseñanza se vean afectadas, ya que dichas creencias se construyen a partir de sus experiencias como alumnos/as.

En la síntesis de Thompson se hace un repaso a la historia de la investigación sobre las creencias del profesorado, en la que señala que es en la década de los ochenta cuando se produce un fuerte resurgimiento de las investigaciones sobre creencias de los/as docentes, con un peso importante de los estudios etnográficos.

A partir de este marco general se han ido sucediendo numerosas investigaciones sobre las creencias del profesorado: unas de carácter más descriptivo y otras centradas en los cambios de dichas creencias, en unas las personas investigadas son docentes en ejercicio mientras que en otras son estudiantes para profesor/a.

En diferentes investigaciones se indican las limitaciones del tratamiento cuantitativo: el cuestionario estandarizado presenta serias limitaciones en relación al significado de los términos (Escudero, García, Llinares y Sánchez, 1993; Llinares, Sánchez, García y Escudero, 1995) e incongruencias entre las diferentes respuestas (Furinghetti, 1994) o entre las respuestas obtenidas y las observaciones realizadas (Foss y Kleinsasser, 1996).

Además, las creencias descritas suelen caracterizarse por una escasa estructuración, pues en un mismo sujeto suelen coexistir diferentes creencias que mantienen muy poca relación entre ellas. Unas veces por las contradicciones del proceso de formación inicial, en el que se transmite teóricamente un modelo de enseñanza pero se practica otro modelo distinto (Blanco, 1996, pp. 206-27), otras por el proceso de aprendizaje recibido en la escuela y que hace que el alumnado opte por una comprensión exclusivamente procedimental (Schoenfeld, 1991). Esto es lo que otros autores denominaron la laguna de la familiaridad (Feiman-Nemser y Buchman, 1988), según ellos la más extendida entre docentes y la menos sujeta a investigación. Ante estas conclusiones, resulta lógico que algunos investigadores centren su atención en los procesos de formación docente, con la idea de analizar qué papel juegan estos procesos en la construcción de las creencias que influirán en su práctica profesional.

Numerosos autores admiten que "los estudiantes para profesor poseen unas concepciones, fruto de sus experiencias personales y sociales, y que estas experiencias están contextualizadas en una determinada cultura escolar" (Sánchez y Llinares, 1996, p. 224). De la misma manera, investigaciones más recientes también plantean que las creencias 
sobre la enseñanza se establecen antes de que el alumnado ingrese a la universidad (de Vries, Van de Grift y Jansen, 2014).

Por eso no es extraño que los/as futuros/as maestros/as piensen que el aprendizaje matemático es mayoritariamente memorístico o mecánico (Caballero, Blanco y Guerrero, 2007) y eso tiene implicaciones directas sobre sus estrategias de enseñanza, ya que la influencia de las creencias del profesorado en sus prácticas del aula suele ser mayor que sus conocimientos (Struyven, Dochy y Janssens 2010; Palos, Ávalos, Flores y Montes, 2017).

Pero la mayoría de las investigaciones toman como objeto de estudio las prácticas docentes de las/os futuras/os profesoras/es, unas veces obviando y otras tomando solo como variables contextuales todas las experiencias escolares anteriores de ese alumnado, pese a que la influencia de esas experiencias aparece explícitamente en los datos obtenidos.

Esta línea de investigación remite al concepto de socialización anticipada (Merton, 1980), que consiste en que las personas adoptan los valores del grupo de referencia, del grupo al que todavía no se pertenece pero al que se aspira. En ocasiones se ha pretendido vincular la socialización anticipada al período de formación universitaria (de la Fuente y Sánchez, 2000), pero la relación causal que se plantea es más hipotética que real y si el grupo de referencia es el de las/os maestras/os, parece lógico que sean las experiencias con sus maestras/os las que configuren esa socialización anticipada, no tanto su relación con el profesorado universitario que no constituye, en ningún momento, el grupo de referencia del alumnado de los Grados de Maestro. Incluso se han señalado las Prácticas de Enseñanza como el comienzo de la socialización profesional del docente (Rodríguez, 2002b), pero sin llegar a determinar si el modelo que se imita es el de las/os maestras/os-tutoras/es o el de sus experiencias escolares anteriores, o si se trata simplemente de una estrategia académica eficaz para el logro de credenciales educativas. En este sentido, Feliu, Vallés y Cardona se sorprenden por "el mayor grado de importancia que los alumnos de cuarto han otorgado a las estrategias de carácter más transmisivo en comparación con los del primer curso" (2016, p. 8), aunque plantean como una posible explicación la influencia de las/os maestras/os-tutoras/es en el modelado de las/os futuras/os docentes.

En los estudios de casos realizados por Swinson y Shield (1994) también se investigaron las experiencias escolares de los sujetos, con el objetivo de averiguar el trabajo en grupo como recurso para cambiar las creencias del alumnado, sin que pudieran concluir que dicho cambio fuese efectivo.

En un trabajo de investigación más amplio —desde 1988 hasta 1991—, sobre un proceso de formación de futuros profesores en la Universidad de Michigan, con una duración de dos años y en el que el alumnado formaba una comunidad de aprendizaje estable - los significados se construyen a partir de la reflexión sobre sus experiencias de enseñanza-, los autores concluyen que "la intervención produce cambios significativos en las creencias de los estudiantes sobre ellos mismos como aprendices de matemáticas, sobre lo que significa conocer matemáticas y sobre la forma de aprender matemáticas" (Wilcox et al., 1991, p. 38), pero esos cambios son fruto de la reflexión compartida y no de un proceso de formación convencional.

Tampoco está claro que en el período de prácticas de las/os estudiantes para maestras/ os se produzcan cambios de creencias. En el trabajo de Sánchez y Llinares (1988) se observaron algunos casos que cambiaron sus creencias pero otros que solo las confirmaron y reforzaron. El problema está en poder determinar el efecto de los cambios de creencias, cuando los hay, en la práctica docente, o si el alumnado adopta prácticas directamente 
relacionadas con sus experiencias escolares anteriores (Bramald, Hardman \& Leat, 1995), ya que una experiencia innovadora aislada "puede ser tan problemática que pueden elegir situarse fuera, y reafirmarse en su forma tradicional de cómo enseñar matemáticas" (Civil, 1996, p. 192).

En la tesis doctoral de P. Flores (1998) se estudia la evolución que sufren las creencias y concepciones del alumnado durante las prácticas de enseñanza, y concluye que las diferencias entre las mediciones pre-test y post-test no son significativas. También incluye un estudio de casos de dos estudiantes, y nuevamente se confirman "las escasas diferencias existentes entre los perfiles elaborados antes y después de las prácticas de enseñanza" (Flores, 1998, p. 243).

Todo esto nos remite a la ya clásica cuestión de la consistencia del sistema de creencias (Green, 1971). Un grado de consistencia de esta envergadura tiene que ser el resultado de un proceso de interiorización que va más allá de cualquier procedimiento instructor, y que podría ser concomitante con el proceso de socialización primaria, en donde se produce un estrecho contacto con la escuela como agente socializador. Las interacciones verticales (docente-alumnado) contribuyen a la interiorización de los roles escolares (Vásquez y Martínez, 1996), y las experiencias (más o menos gratificantes) de cada sujeto serían las que irían dotando de consistencia a los sistemas de creencias que cada alumno/a elabora para, como mínimo, poder desenvolverse en el contexto escolar. En esta línea, W. A. Benítez concluye que "los docentes en formación enseñan de la forma como les enseñan sus docentes, y adquieren muchos de sus modelos, que seguramente forman parte de sus concepciones de la forma de ver su disciplina" (Benítez, 2013, p. 179). La capacidad de interiorización de un proceso tan dilatado en el tiempo es consistente con las investigaciones en las que se muestra que los procesos de formación encaminados a la modificación de la forma de enseñar (como los procesos de actualización docente) tienen muy poca incidencia en la práctica docente inmediatamente posterior (Cristerna, 2019).

\section{DISEÑO DE INVESTIGACIÓN}

\subsection{OBJETIVOS}

El objetivo fundamental es el de describir la influencia del proceso de formación docente, especialmente el período de prácticas, en los sistemas de creencias sobre la enseñanza que tiene el alumnado.

Para ello, además, hay que cubrir el objetivo complementario de describir los sistemas de creencias de las/os futuras/os docentes, analizando las propias trayectorias escolares del alumnado como el lugar donde se desarrollan de dichas creencias. Para delimitar un objeto de estudio que resulta demasiado extenso y complejo, se ha centrado la investigación en las experiencias personales relacionadas con las asignaturas de matemáticas, que están presentes a lo largo de toda la trayectoria escolar de los casos analizados.

\subsection{METODOLOGÍA}

Esta investigación se diseña desde una perspectiva metodológica cualitativa, en donde las técnicas de producción de datos permiten analizar las diferentes visiones y formas de dar 
sentido a las prácticas educativas de los sujetos investigados, y que son las que configuran los sistemas de creencias de cada uno de ellos.

Se proyecta como un estudio de casos de seis estudiantes de la Facultad de Educación de Albacete de la Universidad de Castilla-La Mancha (cuatro del Grado de Maestro en Educación Infantil y dos en Educación Primaria), lo que algunos autores denominan diseño multicaso holístico (Marcelo y Parrilla, 1991, p. 18).

Se trata de una muestra no saturada ya que no se persigue una generalización estadística de los resultados, sino que lo fundamental es observar ejemplos de cómo se forman y desarrollan los sistemas de creencias. Los instrumentos de recogida de datos utilizados han sido:

1. Cuestionario cualitativo ${ }^{1}$ de preguntas abiertas sobre concepciones de la enseñanza, tomado de J. Carrillo (1998, pp. 73-80).

2. Relato libre y escrito sobre las experiencias educativas, en las asignaturas de matemáticas, a lo largo de su vida como estudiante.

3. Dos entrevistas semiestructuradas (una antes y otra después del período de prácticas), con un guión adaptado del utilizado por J. Carrillo (1998, pp. 73-80).

4. Memoria de prácticas, en la que se incluye una valoración personal de la metodología de enseñanza utilizada por el/la tutor/a de cada estudiante.

5. Observación no participante de algunas de las sesiones de clase del período de prácticas del alumnado.

La estrategia básica para la obtención de datos ha consistido en intentar utilizar diferentes técnicas para investigar cuestiones similares. Así, el cuestionario y la primera entrevista se centran en la caracterización del sistema de creencias por parte de cada sujeto y en la descripción de sus experiencias escolares, ya que aún no habían comenzado el período de prácticas y, por tanto, carecían de experiencia docente propia y de los modelos que pudieran experimentar en sus prácticas. Mientras que con la segunda entrevista, la Memoria de Prácticas y la observación directa se pretendía descubrir la práctica docente concreta de cada uno de ellos, de sus maestros/as-tutores/as y la influencia de dichas prácticas en su formación profesional. Lógicamente, estos eran los puntos fundamentales de los guiones de cada una de las técnicas, pero, al tratarse siempre de guiones abiertos, algunos temas pueden aparecer en otros lugares (especialmente el referido a la valoración de su experiencia universitaria).

Esto permite que, para la elaboración de los sistemas de creencias, se puedan comparar los datos obtenidos con el cuestionario y con las entrevistas y asegurar la consistencia de los mismos. Pero también poner en relación el sistema de creencias de cada sujeto con sus experiencias escolares y con el período de prácticas realizado, pues las valoraciones personales que cada sujeto realiza están directamente conectadas con sus propias concepciones sobre la enseñanza.

Para esto se construyen una serie de tablas en donde se representan, para cada sujeto, el sistema de creencias manifestado inicialmente en el cuestionario y la entrevista, así

En sentido estricto, un cuestionario de preguntas abiertas no es ni cuantitativo ni cualitativo, ya que eso sólo depende del tipo de análisis que se haga a partir de la información recogida en el mismo. No obstante, lo adjetivo como cualitativo para que quede claro que las respuestas no se cierran posteriormente para analizarlas estadísticamente, sino que se toman como parte del discurso obtenido de cada sujeto. 
como la relación entre dicho sistema y el conjunto de sus experiencias escolares. Esto permite comparar el sistema de creencias y las experiencias escolares, distinguiendo mediante códigos la tendencia didáctica que cada sujeto conoció durante sus estudios no universitarios y la de su experiencia universitaria en sus estudios de Grado de Maestro.

Para el análisis que se plantea es necesario utilizar una tipología de las tendencias didácticas de la práctica escolar (tabla 1).

Tabla 1. Tendencias didácticas

\begin{tabular}{|c|c|c|c|c|}
\hline Categorías & Tradicional & Tecnológica & Espontaneísta & Investigativa \\
\hline Metodología & $\begin{array}{l}\text { Ejercitación } \\
\text { repetitiva } \\
\text { Programación } \\
\text { oficial } \\
\text { Exposición } \\
\text { magistral (libro de } \\
\text { texto) }\end{array}$ & $\begin{array}{l}\text { Ejercitación } \\
\text { reproductiva } \\
\text { Programación } \\
\text { cerrada } \\
\text { Simulación de } \\
\text { investigación }\end{array}$ & $\begin{array}{l}\text { Experimentación } \\
\text { Programación } \\
\text { negociada } \\
\text { Descubrimiento } \\
\text { aleatorio }\end{array}$ & $\begin{array}{l}\text { Investigación } \\
\text { planificada } \\
\text { Red conceptual } \\
\text { organizada } \\
\text { Investigación } \\
\text { planificada }\end{array}$ \\
\hline Asignatura & $\begin{array}{l}\text { Conceptual } \\
\text { (proceso) } \\
\text { Finalidad } \\
\text { informativa }\end{array}$ & $\begin{array}{l}\text { Aplicabilidad } \\
\text { (proceso- } \\
\text { producto) } \\
\text { Finalidad utilitaria }\end{array}$ & $\begin{array}{l}\text { Procedimental y } \\
\text { actitudinal } \\
\text { Formativa } \\
\text { (racional) }\end{array}$ & $\begin{array}{l}\text { Procedimientos, } \\
\text { conceptos y } \\
\text { actitudes } \\
\text { Formativa } \\
\text { (aprender a } \\
\text { aprender) }\end{array}$ \\
\hline Aprendizaje & $\begin{array}{l}\text { Memorístico } \\
\text { acumulativo } \\
\text { Por apropiación } \\
\text { Trabajo individual }\end{array}$ & $\begin{array}{l}\text { Memorístico } \\
\text { secuencial } \\
\text { Por asimilación } \\
\text { Trabajo individual }\end{array}$ & $\begin{array}{l}\text { Significativo } \\
\text { aleatorio } \\
\text { Por construcción } \\
\text { espontánea } \\
\text { Trabajo en grupo/ } \\
\text { debates }\end{array}$ & $\begin{array}{l}\text { Significativo } \\
\text { relevante } \\
\text { Por construcción } \\
\text { dirigida } \\
\text { Diversidad de } \\
\text { agrupaciones y } \\
\text { puestas en común } \\
\end{array}$ \\
\hline $\begin{array}{l}\text { Papel del } \\
\text { alumnado }\end{array}$ & $\begin{array}{l}\text { No participa } \\
\text { Escucha y copia } \\
\text { Atiende y acepta }\end{array}$ & $\begin{array}{l}\text { No participa } \\
\text { Reproduce e imita } \\
\text { Atiende y cree }\end{array}$ & $\begin{array}{l}\text { Participación } \\
\text { indirecta } \\
\text { Actúa } \\
\text { Juega y dialoga }\end{array}$ & $\begin{array}{l}\text { Participación } \\
\text { directa } \\
\text { Investiga } \\
\text { Reflexiona y } \\
\text { cuestiona }\end{array}$ \\
\hline $\begin{array}{l}\text { Papel del } \\
\text { profesorado }\end{array}$ & \begin{tabular}{|l|} 
Transmite \\
verbalmente \\
Dicta y reproduce
\end{tabular} & $\begin{array}{l}\text { Transmite por } \\
\text { procesos } \\
\text { tecnológicos } \\
\text { Expone y organiza }\end{array}$ & $\begin{array}{l}\text { Induce } \\
\text { Promueve } \\
\text { Analiza las } \\
\text { reacciones }\end{array}$ & $\begin{array}{l}\text { Provoca } \\
\text { Conduce } \\
\text { Investiga sobre la } \\
\text { acción }\end{array}$ \\
\hline Evaluación & $\begin{array}{l}\text { Sumativa } \\
\text { Controles del } \\
\text { producto } \\
\text { Criterios rígidos }\end{array}$ & $\begin{array}{l}\text { Sumativa } \\
\text { Controles de los } \\
\text { objetivos } \\
\text { Criterios rígidos } \\
\text { modificables }\end{array}$ & $\begin{array}{l}\text { Formativa } \\
\text { Revisión de las } \\
\text { tareas del alumno } \\
\text { y su participación } \\
\text { Criterios } \\
\text { negociables }\end{array}$ & $\begin{array}{l}\text { Formativa } \\
\text { Por conjunción de } \\
\text { varios } \\
\text { instrumentos } \\
\text { Criterios } \\
\text { reformulables }\end{array}$ \\
\hline
\end{tabular}


Se trata de un esquema analítico en el que poder situar las creencias de los sujetos analizados, y aquí se ha utilizado la terminología empleada por R. Porlán (1989) y la reelaboración realizada por J. Carrillo (1998), en donde se distingue entre enseñanza tradicional, tecnológica, espontaneísta e investigativa, según una serie de variables relacionadas sobre la metodología docente (Praxis, Objetivos, Programación), el sentido de la asignatura (Orientación, Finalidad), la concepción del aprendizaje (Tipo y forma, Tipo de agrupamiento, Dinamizador), el papel del alumnado (Participación en el diseño didáctico, Clave de la transferencia enseñanza-aprendizaje, ¿Qué hace?), el papel del profesorado (¿Qué hace?, ¿Cómo hace?) y el proceso de evaluación (Carácter, Criterios, Tipo de calificación). Aunque existen claras diferencias entre cada una de estas tendencias didácticas, también existen puntos en común entre ellas, de manera que las dos primeras (Tradicional y Tecnológica) responden al modelo de educación bancaria propuesto por Freire, en la que "el único margen de acción que se ofrece a los educandos es el de recibir los depósitos, guardarlos y archivarlos. Margen que sólo les permite ser coleccionistas o fichadores de cosas que archivan" (2005, p. 78), ya que ambas funcionan mediante la ejercitación y el aprendizaje memorístico de unos saberes que el alumnado es incapaz de construir. Sin embargo, las tendencias Espontaneísta e Investigativa trabajan mediante la experimentación del alumnado para desarrollar aprendizajes significativos. Utilizando el texto de Carrillo se puede presentar un resumen de las cuatro tendencias didácticas.

\section{RESULTADOS}

Los perfiles obtenidos en el análisis de tendencias didácticas, pese a que el conjunto de creencias que se recogen mediante el cuestionario y la primera de las entrevistas es, sobre todo, de carácter espontaneísta (excepto en las categorías correspondientes al sentido de la asignatura de matemáticas, en donde la tendencia tecnológica es la más frecuente), presentan diferencias importantes en cada uno de los sujetos. Lógicamente, las tendencias son construcciones teóricas que facilitan el análisis, pero ningún caso se ajusta por completo a cualquiera de ellas.

\subsection{NADIA}

Aquí aparecen referencias de cada una de las cuatro tendencias didácticas. Se trata del perfil que menos se ajusta a cualquiera de los modelos preestablecidos y que, aunque la tendencia espontaneísta es la que obtiene un mayor recuento, es en el que más aparece la tendencia tradicional. Tanto en la metodología (especialmente en la praxis), como en el papel del alumnado y la evaluación, se mantienen algunos de los principios básicos de la concepción bancaria de la educación.

Este discurso más tradicional (en cuestiones de fondo) se equilibra, casi exclusivamente, por las referencias a la utilización de recursos y materiales didácticos, y a una concepción del aprendizaje que se puede denominar como constructivismo espontáneo (cuestiones de forma).

Solamente en el ámbito del papel del docente es en donde el perfil se sitúa entre el tecnologismo y el espontaneísmo, tal vez porque la formación universitaria esté contribuyendo al proceso de socialización profesional anticipada. 
Entre los recuerdos de su experiencia escolar de las matemáticas destaca una mecánica pedagógica determinada (explicación-ejemplos-problemas). Esta mecánica (que se puede considerar tradicional, al menos en el sentido de que, espacial y temporalmente, tiene una presencia destacada en la historia de la escuela española) genera, entre otras cosas, la sensación de seguridad en la alumna: después de una explicación lo más concreta posible, se realizan ejercicios que la proveen de una serie de destrezas para resolver ese tipo de ejercicios. En la medida en que ese tipo de ejercicios son los que tendrá que resolver en el examen, ella siente la seguridad de que no tendrá problemas para superar la asignatura.

De ahí que a Nadia le guste que el trabajo docente consista en guiar al alumnado con una dinámica similar, por lo que su perfil se mantiene entre lo tradicional (en el fondo) y lo espontaneísta (en la forma) (tabla 2)².

Tabla 2. Sistema de creencias y perfiles biográficos de Nadia

\begin{tabular}{|c|c|c|c|c|c|}
\hline Categorías & Tradicional & Tecn & gica & Espontaneísta & Investigativa \\
\hline Metodología & - 0 & & & - & \\
\hline Sentido de la asignatura & & - $c$ & $x$ & $\bullet$ & \\
\hline Concepción del aprendizaje & & $c$ & $x$ & 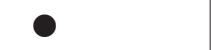 & \\
\hline Papel del alumno & 0 & & $x$ & - & \\
\hline Papel del profesor & & - & $x$ & $\bullet$ & \\
\hline Evaluación & 0 & & & ? & \\
\hline
\end{tabular}

- Sistema de creencias $\mathrm{O}=$ Experiencia escolar $\mathrm{X}=$ Experiencia universitaria.

\subsection{ANA}

Este caso se ajusta bastante a la tendencia espontaneísta (siempre con la excepción señalada del sentido de la asignatura de matemáticas), pero que todavía mantiene creencias tradicionales respecto a la praxis de la metodología didáctica.

La repetición de ejercicios y la exposición magistral coinciden aquí con la experimentación y el descubrimiento aleatorio por parte del alumnado, de manera que, como en el caso anterior, existe una cierta disonancia entre una metodología de enseñanza basada en principios bancarios pero con el añadido espontaneísta del uso de recursos didácticos y de actividades variadas.

\footnotetext{
En estas tablas se representan las tendencias generales obtenidas tras el análisis. Los tres códigos utilizados se corresponden con el sistema de creencias $(\bullet)$, que es una representación simplificada a partir de las 16 variables de la tipología utilizada por J. Carrillo (1998); la experiencia escolar (O), que se refiere a la tendencia didáctica que cada sujeto conoció durante sus estudios no universitarios, y la experiencia universitaria $(X)$ que es la que han tenido en sus estudios de Grado de Maestro. En algunos casos (Ana, María, Llanos y Carmen), los perfiles biográficos presentan dos tipos de experiencias escolares diferentes. En ese caso, la tendencia didáctica que haya sido minoritaria (en términos de cantidad) se representa mediante círculos de menor tamaño. Esta distinción obedece sólo a razones técnicas y no indica una mayor o menor importancia de dichas experiencias en relación con el sistema de creencias obtenido de cada sujeto, pero es necesario señalarla, pues supone un cierto conflicto en algunos aspectos del proceso socializador que tiene lugar en la escuela (al menos en lo que respecta al aprendizaje de los roles de maestra/o y de alumna/o y a la relación pedagógica que se establece entre ambos).
} 
Los recuerdos de sus experiencias escolares se ajustan al modelo de educación bancaria, señalando explícitamente alguno de sus pilares fundamentales: la autoridad del docente como marco fundamental de la relación pedagógica, la memoria como destreza básica para el proceso de aprendizaje y la repetición como estrategia para la acumulación de conocimientos. Y esto se asocia también con la idea de aburrimiento escolar, que en algunos casos puede adoptar la forma de frustración.

Pero junto a la crítica explícita de su experiencia escolar también manifiesta una cierta incapacidad profesional para intentar afrontar el trabajo docente de otro modo. Parece que su período de formación universitaria sólo le ha servido para conocer recursos y materiales para utilizar en las clases, pero serán clases que seguirán reproduciendo un proceso de enseñanza y aprendizaje muy similar al que ella conoció como alumna.

Se trataría de un intento de renovación tecnológica (actividades, recursos, juegos) de la misma dinámica escolar, de ahí que tengan que ser esos elementos tecnológicos los que añadan la motivación (elemento central en su formación universitaria) de la que carecía cuando ella estudiaba, y que puede estar relacionada con algunas breves experiencias acumuladas en sus estudios universitarios, en las que se utilizaba una metodología diferente a la más tradicional, pero de la que destaca especialmente la utilización de recursos y actividades diversas.

Pero esta solución renovadora resulta tan débil, que la propia alumna reconoce que son sus experiencias previas las que utilizará en su ejercicio profesional (con el añadido de lo que pueda ver en las Prácticas de Enseñanza, que acaba siendo más de lo mismo, es decir, una forma de reforzar un sistema de creencias que reproduce la relación pedagógica tradicional) (tabla 3).

Tabla 3. Sistema de creencias y perfiles biográficos de Ana

\begin{tabular}{|c|c|c|c|c|c|c|}
\hline Categorías & \multicolumn{2}{|c|}{ Tradicional } & \multirow[t]{2}{*}{ Tecnológica } & \multicolumn{2}{|c|}{ Espontaneísta } & Investigativa \\
\hline Metología & - 0 & $x$ & & $\bullet$ & $x$ & \\
\hline Sentido de la asignatura & & & $\bullet$ & & & \\
\hline Concepción del aprendizaje & O & $x$ & & $\bullet$ & & \\
\hline Papel del alumno & o & $x$ & & $\bullet$ & $x$ & \\
\hline Papel del profesor & O & $x$ & & $\bullet$ & $x$ & \\
\hline Evaluación & O & & & $\bullet$ & & \\
\hline
\end{tabular}

- Sistema de creencias $\mathrm{O}=$ Experiencia escolar $\mathrm{X}=$ Experiencia universitaria.

\subsection{MARÍA}

Las creencias manifiestas de María se sitúan en las tendencias espontaneísta y tecnológica. Esta segunda en las variables del sentido de la asignatura de matemáticas y en el papel del profesor. 
Esto hace que las creencias manifiestas se centren en aspectos específicamente metodológicos pero que, de manera implícita, se manejen los conceptos de jerarquía y autoridad en la figura del docente, manteniendo así una cierta concepción bancaria de la educación, similar a la de los dos casos anteriores.

El resumen de las experiencias escolares de María, en las asignaturas de matemáticas, hasta el $2^{\circ}$ curso de E.S.O. es el de la rutina del aburrimiento. En este punto es un caso muy similar al de Ana, pues se repite la dinámica de explicaciones del profesor y extensa reiteración de ejercicios.

Pero en este caso existe una diferencia importante: durante el bachillerato experimenta una manera diferente de enseñar matemáticas. Se trata de un Taller de matemáticas, en donde la rutina desaparece al plantearse problemas no estandarizados. Esta misma experiencia se repite en sus estudios universitarios, en donde se añade el conocimiento de diferentes recursos y materiales didácticos relacionados con la enseñanza de las matemáticas, y que refuerza su propio sistema de creencias.

En su perfil no aparecía ningún elemento de la tendencia tradicional. Esto hace que en las Prácticas de enseñanza, en donde su tutor sí que plantea las clases mediante la rutina que ella criticó al hablar de su pasado escolar, se produzca una divergencia entre los modelos de enseñanza de uno y otra (tabla 4).

Tabla 4. Sistema de creencias y perfiles biográficos de María

\begin{tabular}{|c|c|c|c|c|c|c|}
\hline Categorías & Tradicional & Tecnológica & Esp & nta & eísta & Investigativa \\
\hline Metodología & $0 \times$ & & - & 0 & $x$ & - \\
\hline Sentido de la asignatura & O & $\bullet$ & $\bullet$ & & & \\
\hline Concepción del aprendizaje & 0 & & $\bullet$ & 0 & & \\
\hline Papel del alumno & $0 \times$ & & $\bullet$ & 0 & $x$ & \\
\hline Papel del profesor & $0 \times$ & $\bullet$ & & 0 & $x$ & \\
\hline Evaluación & O & & - & & & \\
\hline
\end{tabular}

- Sistema de creencias $\mathrm{O}=$ Experiencia escolar $\mathrm{X}=$ Experiencia universitaria

\subsection{ARES}

El perfil de este alumno es muy parecido al de María (de orientación espontaneísta excepto en las variables del sentido de la asignatura y del papel del docente), pero mientras que María adoptaba para el papel del docente una tendencia tecnológica, Ares se aproxima más a los términos de la tendencia investigativa.

No es que plantee un modelo de enseñanza netamente constructivista (ni realiza una crítica clara del modelo tradicional ni opta por otro modelo concreto), sino que se queda en el nivel de la metodología didáctica, con un énfasis especial en la participación del alumnado en numerosas actividades y con un alto grado de empatía entre alumnado y docente. 
Este caso presenta una particularidad frente a los demás: las valoraciones (positivas y negativas) que hace sobre su pasado escolar no tienen una vinculación directa con los métodos de enseñanza de las matemáticas en sentido estricto, sino que las realiza en función del tipo de sentimientos (feeling) establecidos entre alumnado y docente. Los docentes simpáticos hacían sus clases entretenidas, los que no las hacían entretenidas le caían mal. Pero la metodología didáctica era muy similar, y consistía en el esquema explicaciónejercicios en cada uno de los temas.

Realmente no plantea una crítica pedagógica a un modelo didáctico determinado, ni parece decantarse claramente por ninguno en particular. Es cierto que su perfil se sitúa entre las tendencias espontaneísta e investigativa, pero mantiene la idea tradicional del profesor como transmisor de conocimientos a los alumnos.

Pero al hablar de las Prácticas de enseñanza introduce una valoración diferente a la que realiza sobre su pasado escolar. Ahora se ha vuelto a encontrar la dinámica pedagógica que consiste en la serie explicación-ejercicios (que en Educación Infantil adoptan la forma de fichas), pero ahora la cataloga directamente como poco activa, aburrida, y aboga por cambios metodológicos hacia lo que denomina métodos más humanos. Reconoce que es lo mismo que él conoció como alumno, pero pensaba que el paso del tiempo implicaría una modificación del trabajo pedagógico.

Y al finalizar dicho período de Prácticas, todo esto se resume en que, aunque lo critique, el modelo de enseñanza tradicional que él conoció como alumno es el único del que dispone como futuro maestro. Y cuando se le plantean situaciones en el aula en las que se siente inseguro, busca esa seguridad en lo que criticó como alumno: la autoridad del profesor y la repetición sistemática de fichas y ejercicios (tabla 5).

Tabla 5. Sistema de creencias y perfiles biográficos de Ares

\begin{tabular}{|l|c|c|c|c|}
\hline \multicolumn{1}{|c|}{ Categorías } & Tradicional & Tecnológica & Espontaneísta & Investigativa \\
\hline Metodología & $\circ \times$ & & $\bullet$ & \\
\hline Sentido de la asignatura & & $\bullet$ & & \\
\hline Concepción del aprendizaje & & $\circ$ & $\bullet$ & \\
\hline Papel del alumno & & $\circ$ & $\bullet$ & \\
\hline Papel del profesor & $\times$ & $\circ$ & $\bullet$ & $\bullet$ \\
\hline Evaluación & & $\circ$ & $\bullet$ & \\
\hline
\end{tabular}

- Sistema de creencias $\mathrm{O}=$ Experiencia escolar $\times=$ Experiencia universitaria.

\subsection{LLANOS}

Este perfil se diferencia de todos los demás. La tendencia espontaneísta es claramente dominante (incluso en el sentido de la asignatura), sin apenas interferencias de las otras tendencias, aunque también expresa la tendencia investigativa en casi todas las categorías. 
En cualquier caso, hay que señalar que aquí pueden estar interviniendo otras variables, pues forma parte de una amplia red familiar con vinculaciones profesionales con la docencia, de manera que su potencial socialización anticipada puede haberse producido en ámbitos sociales que no forman parte de esta investigación.

En las experiencias de Llanos se relatan dos formas diferentes de trabajo docente. La que denomina como enseñanza por descubrimiento (con un esquema general basado en el binomio acción-reflexión) que, más o menos idealizada, siempre presenta unas valoraciones muy positivas, y otra más tradicional (organizada alrededor de los valores de jerarquía, autoridad, disciplina y sumisión) con valoraciones muy negativas (según esta alumna, esa es la causa de su especial aversión hacia las matemáticas).

Este posicionamiento favorable a discursos constructivistas (aunque con una escasísima teorización) también se refleja en su perfil de creencias, con una posición dominante de la tendencia espontaneísta y el añadido de algunas variables de la tendencia investigativa.

En sus Prácticas de enseñanza, inicialmente está con una maestra que no se ajusta a su idea del trabajo docente, pero eso parece no afectar a su sistema de creencias. Es más, cuando después, con otra maestra, observa que mucho de lo que ella piensa se puede realizar en el aula, refuerza sus creencias, señalando que con esa doble experiencia en sus Prácticas de enseñanza ha tenido la ventaja de poder observar diferentes formas de trabajar en el aula y afianzar tanto lo que quiere hacer como lo que no quiere hacer cuando ella trabaje como maestra (tabla 6).

Tabla 6. Sistema de creencias y perfiles biográficos de Llanos

\begin{tabular}{|c|c|c|c|c|c|c|}
\hline Categorías & Tradicional & Tecnológica & \multicolumn{3}{|c|}{ Espontaneísta } & Investigativa \\
\hline Metodología & $x$ & & & 0 & $x$ & \\
\hline Sentido de la asignatura & & & $\bullet$ & & & \\
\hline Concepción del aprendizaje & $x$ & & $\bullet$ & O & $x$ & \\
\hline Papel del alumno & $x$ & & $\bullet$ & O & $x$ & • \\
\hline Papel del profesor & $x$ & & $\bullet$ & O & $x$ & $\bullet$ \\
\hline Evaluación & & & $\bullet$ & & & \\
\hline
\end{tabular}

- Sistema de creencias $\quad \mathrm{O}=$ Experiencia escolar $\quad \mathrm{X}=$ Experiencia universitaria.

\subsection{CARMEN}

Nuevamente se trata de una alumna que se sitúa en la tendencia espontaneísta, pero aquí es donde hay una presencia mayor de creencias investigativas, especialmente con respecto a los papeles del alumnado y del docente.

La opción por una metodología basada en la participación de todos conlleva una relación pedagógica diferente al modelo tradicional habitual. 
Aquí también hay algunas variables externas importantes: se trata de la alumna de mayor edad (tres años más que en los otros casos) y con un período mayor de formación escolar (incluido un módulo de Formación Profesional, en donde se trabajan más los aprendizajes prácticos). También aparece un claro sentido vocacional en su elección de los estudios de Maestro, y manifiesta que es antes de su entrada en la universidad cuando ya ha hecho no sólo una elección profesional, sino una elección por una determinada manera de ejercer esa profesión.

Como en el caso anterior, Carmen señala que en sus experiencias escolares ha observado dos modelos diferentes de plantear el trabajo pedagógico. Uno con métodos activos y prácticos (y en los que, según ella, se concede gran importancia a las relaciones afectivas), que en su biografía se corresponden con la Educación Infantil y con un Módulo de Formación Profesional, y otro modelo más teórico y alejado de la práctica cotidiana de las/os alumnas/os, que es el que conoció en la Educación Primaria y el Bachillerato.

Y también, como en el caso de Llanos, presenta el perfil más definido alrededor de la tendencia espontaneísta, con algunos elementos de la tendencia investigativa (en algún momento llega a plantear la importancia de la reflexión en la acción para el trabajo docente). Ella misma señala que esas experiencias son las que le han servido para elaborar su sistema de creencias sobre la enseñanza, y que la elaboración de ese sistema es anterior a su período de formación universitaria (aquí también se incluye una crítica directa al modelo universitario de formación docente, pues considera esta formación mucho menos operativa que la que conoció en el módulo de Formación Profesional).

Así, las Prácticas de enseñanza no han supuesto ningún cambio en sus creencias. Ha habido algunos aspectos del trabajo de la maestra en los que coincidía con lo que piensa Carmen (especialmente la metodología activa y el diálogo con el alumnado), pero también otras cuestiones con las que discrepa abiertamente (el bajo nivel de afectividad de la maestra con el alumnado y el exceso de trabajo con fichas).

En cualquier caso, ella no es consciente de que haya cambiado su forma de pensar al respecto pues, además, las clases que ella ha dirigido, según su sistema de creencias, se han desarrollado sin problemas y la evaluación que realiza es bastante positiva (tabla 7).

Tabla 7. Sistema de creencias y perfiles biográficos de Carmen

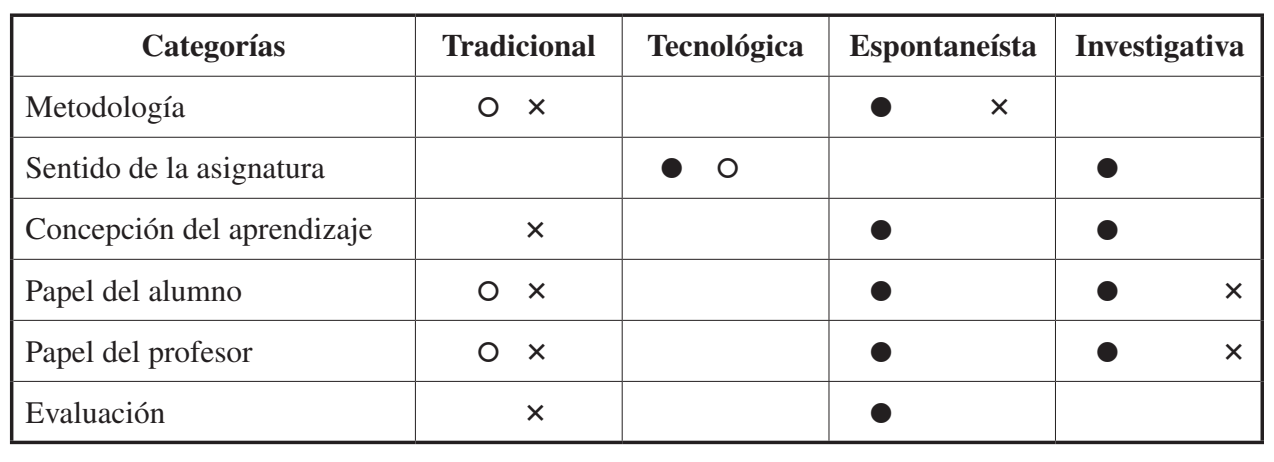

- Sistema de creencias $\mathrm{O}=$ Experiencia escolar $\quad \times=$ Experiencia universitaria. 


\section{DISCUSIÓN}

De estos perfiles, variados por la heterogeneidad de los sujetos, pero con algunas regularidades evidentes, se pueden extraer algunas consideraciones sobre los sistemas de creencias y la formación profesional del maestro.

En primer lugar, destaca la generalización de la tendencia espontaneísta, especialmente en las categorías de metodología, papel del alumnado y papel del docente, y centrándose la mayor parte de las veces en los aspectos procedimentales de dicha tendencia didáctica. El conjunto de situaciones, actividades y modelos que se presentan al alumnado es algo central en los discursos de los casos analizados, pues no sólo constituyen la dinámica de las clases (tal y como las piensan en su futuro profesional), sino que también son el fundamento de la motivación y el aprendizaje del alumnado, sin llegar a argumentar un modelo teórico de dicho aprendizaje que sustente esos planteamientos metodológicos. En algunos momentos parece que el trabajo docente se reduce a mantener actividades y dinámicas de grupo variadas y entretenidas, con un especial temor al aburrimiento infantil, esperando que, desde ahí, cada alumno sea capaz de construir su propio conocimiento.

En segundo lugar, los diferentes perfiles obtenidos tienen diferentes niveles de definición. Desde el de Nadia (el más difuso en el sentido de que se ajusta menos a una sola tendencia), hasta los de Carmen y Llanos (en los que hay menos dispersión en sus elecciones y, por tanto, ofrecen un mayor nivel de consistencia). Para obtener conclusiones válidas en este terreno sería conveniente incluir más casos en el estudio, pero parece apuntarse que, en la medida en que el perfil se aleja del modelo tradicional, este tiende a estar más definido (centrado en una tendencia, o dos a lo sumo) y, por tanto, con menos contradicciones internas.

En tercer lugar, la escasa implantación del modelo constructivista de enseñanzaaprendizaje, pues se defiende un modelo mucho más mecánico y memorístico, al igual que ocurría en el estudio realizado en la Universidad de Extremadura (Caballero, Blanco y Guerrero, 2007).

Aparentemente, el constructivismo domina los planes de formación de docentes de las últimas décadas, al menos si nos fijamos en los programas y las bibliografías de buena parte de las asignaturas de los planes de estudio. No en vano es el modelo que se consagra tras la reforma educativa producida por la L.O.G.S.E., y a partir del cual comienzan a constituirse en España los campos académicos de las diferentes didácticas especiales, pero en este trabajo se confirma que la influencia de las creencias supera a la de los conocimientos (Struyven, Dochy y Janssens, 2010; Palos, Ávalos, Flores y Montes, 2017).

Se puede argumentar que el Grado de Maestro está diseñado desde una perspectiva esencialmente técnico-práctica ${ }^{3}$, por lo que carece de una mínima reflexión epistemológica sobre el proceso de enseñanza-aprendizaje, lo que explicaría que las/os futuras/os docentes

Utilizando la tipología desarrollada por A. Pérez (1992), la planificación de la formación docente desarrollada en las Facultades de Educación responde a una mezcla de las perspectivas técnica y práctica: en el primer caso porque se pretende la formación de un profesional (técnico) con capacidad fundamentalmente instrumental en el terreno de las habilidades de intervención pedagógica, aunque dentro del modelo que Pérez Gómez denomina de adopción de decisiones; y en el segundo caso porque se concibe al docente "como un artesano, artista o profesional clínico que tiene que desarrollar su sabiduría experiencial y su creatividad para afrontar las situaciones únicas, ambiguas, inciertas y conflictivas que configuran la vida del aula" (Pérez, 1992, p. 410). 
sean, en su mayoría, incapaces de plantear un modelo que explique las estrategias de enseñanza que planean.

Cuando, además, una parte importante de los créditos del Grado se planifican para impartir el modelo constructivista, resulta más sensato empezar a reflexionar sobre el impacto real de esta enseñanza universitaria, y cuál es el papel que juega en el proceso de socialización profesional. La elección espontaneísta que, en mayor o menor medida, está presente en estos estudiantes puede ser entendida como una vulgarización del constructivismo que parece presidir el currículum explícito de su período de formación docente, lo que supone que dicho currículum no se desarrolla como aparece diseñado, o que tiene un escaso impacto frente a otros factores menos explícitos, o ambas cosas a la vez.

En cuarto lugar, la crítica generalizada a ciertas estrategias pedagógicas universitarias. Al margen de los contenidos explícitos de sus estudios universitarios, los sujetos manifiestan con claridad que las estrategias pedagógicas que se han llevado a cabo en dichos estudios presentan grandes diferencias con los planteamientos teóricos que se impartían en esas mismas asignaturas. La percepción que ellos tienen es la de una enseñanza universitaria exclusivamente memorística, de transmisión de conocimientos teóricos por parte del profesor, muy próxima a lo que P. Freire catalogó como concepción bancaria de la educación, y que está en contradicción con los propios contenidos que se están transmitiendo.

Esta disonancia entre el contenido y la metodología en sus estudios universitarios tiene efectos en el proceso de socialización docente. En todos los casos incluidos en esta investigación coincide la crítica a la metodología bancaria de su aprendizaje universitario con la adhesión a la tendencia espontaneísta (esto es especialmente claro en los aspectos metodológicos), de ahí que no resulte arriesgado establecer dicha disonancia como uno de los ejes que estructuran las creencias sobre la enseñanza de las/os futuras/os maestras/ os. La oposición entre conocimiento práctico y conocimiento teórico se presenta en estos estudiantes como oposición entre aprendizaje y enseñanza, entre actividad y pasividad, entre lo entretenido y lo aburrido.

Hasta ahora se ha visto que no existe una relación directa entre el currículum explícito de la formación de docentes y los sistemas de creencias sobre la enseñanza del alumnado. Y también parece claro que, en los casos estudiados, las estrategias pedagógicas dominantes en la Facultad de Educación suelen servir como muestra de lo que las/os alumnas/os no deben hacer cuando trabajen como docentes. Pero esto deja dos importantes interrogantes sin resolver: ¿a partir de qué experiencias socializadoras se construyen esos sistemas de creencias?, y ¿qué tipo de influencia ejercen las Prácticas de Enseñanza en esas creencias?

En este sentido, entre los instrumentos de recogida de datos se incluyeron los relatos sobre sus experiencias educativas en clases de matemáticas durante toda su trayectoria escolar, así como también se realizó una segunda entrevista en todos los casos, en la que se incluía una valoración de las Prácticas de enseñanza (que contenía preguntas sobre el trabajo desarrollado por las/os maestras/os, el trabajo desarrollado por las/os alumnas/os, y su propia valoración sobre el impacto de dichas Prácticas en sus creencias personales). La intención era, en primer lugar, la de comprobar si se puede establecer algún tipo de relación entre su proceso de socialización en la escuela y su sistema de creencias sobre la enseñanza, y en segundo lugar observar qué tipo de influencia ejerce el período de las Prácticas de enseñanza en dichas creencias. Tal y como ocurría al observar los perfiles trazados para cada sujeto, al establecer una relación entre experiencias escolares, perfil 
de creencias y período de Prácticas de enseñanza, se observa, como mínimo, una serie de regularidades en todos los casos estudiados:

Todos los perfiles se corresponden, en cada caso, con las experiencias escolares más gratificantes que recuerdan. Tanto quienes se sitúan en una tendencia más tradicional, como quienes lo hacen en el modelo espontaneísta, lo hacen en unos términos muy similares a los que señalan al hablar de sus recuerdos escolares, lo que confirma que sus sistemas de creencias se desarrollan antes de su formación universitaria (de Vries, Van de Grift y Jansen, 2014).

Solamente en los casos de Nadia y Ana, en donde su experiencia escolar se reduce exclusivamente al modelo tradicional, se observa una variación en sus perfiles en la medida en que optan por ciertos planteamientos espontaneístas (especialmente en cuestiones referidas a la dinámica de las clases, con la idea de escapar del aburrimiento que ellas conocieron, para poder, en palabras de Ana, motivar al alumnado), pero siempre sin que ello sirva para poner en cuestión el modelo educativo que conocieron como alumnas (y que presenta las características básicas de la concepción bancaria de la educación).

La experiencia de la asignatura Prácticas de enseñanza no modifica los sistemas de creencias de los seis casos estudiados, tal y como también se señala en otras investigaciones (Flores, 1998; Benítez, 2013).

En los casos de Nadia y Ana, la experiencia de las prácticas no ha supuesto ningún conflicto entre sus creencias y el trabajo pedagógico desarrollado por su maestra-tutora, mientras que en los otros cuatro casos (en los que adoptan diferentes perfiles espontaneístas) sí existen diferencias entre los planteamientos iniciales de los sujetos y la dinámica escolar gestionada por la maestra-tutora. Pero en los cuatro casos, en lugar de que esto provoque una modificación de sus creencias, refuerzan dichos sistemas, en contraposición a los modelos más tradicionales que les recordaban a los que ellos tuvieron en su paso por la escuela y el instituto.

Es cierto que, en los seis casos incluidos en este trabajo, la experiencia de las Prácticas de enseñanza ha sido muy similar. Esto ha significado que las alumnas con un perfil más tradicional no hayan visto otra forma de trabajo pedagógico que la que ya conocían desde su infancia. Es plausible que, en el caso de Ana, sí se podrían modificar esas creencias, en la medida en que ella misma reconocía no estar satisfecha con ese tipo de trabajo pedagógico, pero, al mismo tiempo, se sentía incapacitada profesionalmente para intentar desarrollarlo de otra manera.

Finalmente, se puede afirmar que las creencias sobre la enseñanza se generan en los procesos de socialización que tienen lugar a lo largo de toda la vida escolar del alumnado, sin que el período de formación estrictamente docente tenga una mayor influencia que cualquier otra etapa escolar.

En relación con lo anterior, cualquier intento de modificación de los sistemas de creencias sobre la enseñanza tiene que partir del aprendizaje práctico. Aquí, aprendizaje práctico se refiere a un proceso de interiorización a partir de diferentes experiencias prácticas, fruto del trabajo de reflexión sobre dichas prácticas pedagógicas, pero esto es entrar ya en el terreno de las conclusiones finales de la investigación. 


\section{CONCLUSIONES}

En relación con los sistemas de creencias de los futuros docentes, aunque no se puede concluir si es debido al currículum de los estudios universitarios del Grado de Maestro, los sistemas de creencias sobre la enseñanza son, utilizando la terminología adoptada por Carrillo, fundamentalmente espontaneístas.

Probablemente, en estos modelos espontaneístas están interviniendo dos variables importantes: en primer lugar, el agotamiento de los modelos tradicionales, en el sentido de que el propio alumnado conoce sus limitaciones (relacionadas con el aburrimiento escolar), aunque también conoce las ventajas de la comodidad laboral (que siempre están presentes en los procesos de acomodación del profesorado) y, en segundo lugar, la presencia de las didácticas específicas en los planes de estudio de los Grados de Maestro, de las que las/os alumnas/os captan con facilidad el catálogo de recursos didácticos pero son incapaces de formular un modelo pedagógico que sustente prácticas escolares alejadas de la concepción bancaria de la educación.

Esto puede explicar que no se produzca el paso del espontaneísmo al constructivismo. Pese a que las reformas educativas iniciadas en España durante la década de los ochenta eran, sobre el papel, fruto de las teorías constructivistas del aprendizaje, los procesos de formación docente asociados a dichas reformas no han conseguido que dichas teorías sean la base del pensamiento de los futuros docentes.

Sobre el origen y el proceso de formación de estos sistemas de creencias, se confirma que guardan una relación directa con las experiencias escolares. No se puede interiorizar como sistema de creencias algo que no se haya aprendido mediante un proceso de socialización. Y los procesos de socialización son lo suficientemente dilatados en el tiempo como para pretender que el escaso período de formación universitaria juegue un papel más importante que el resto de las experiencias escolares del alumnado, máxime cuando algunas de esas experiencias han tenido lugar a lo largo del proceso de socialización primaria. Las creencias, como los valores, no se aprenden mediante procesos instructores sino mediante procesos socializadores.

Unido a esto, en la medida en que un/a futuro/a docente haya tenido una mayor diversidad de experiencias escolares (en el sentido de diferentes dinámicas pedagógicas, que es el que se está utilizando a lo largo de todo este trabajo), tiene más elementos para poder configurar un sistema de creencias propio que, por esa misma razón, será un sistema de creencias mucho más definido (más consistente) que quienes hayan carecido de esa diversidad.

Todo esto tiene consecuencias directas en los procesos de formación docente: si se quieren fomentar unos sistemas de creencias determinados, sólo se puede realizar desde la práctica pedagógica (no desde la instrucción formal del programa de cualquier asignatura pedagógica). Y, como medida cautelar, siempre es preferible que las experiencias acumuladas por futuros docentes sean lo más diversas posibles, no solo como punto de partida para un proceso de reflexión profesional docente, sino para prevenir el riesgo de la uniformidad. Al fin y al cabo, cada docente (universitario) desarrolla su trabajo según su sistema de creencias (y con ello las transmite), de manera que para intentar escapar del círculo de reproducción pedagógica hay que situarse en el terreno de la diversidad.

En el terreno más concreto del período de Prácticas del proceso de formación universitaria, se ha visto que su nivel de influencia es más bien escaso. Habitualmente 
estas Prácticas de enseñanza operan como refuerzo de los sistemas de creencias de las/os alumnas/os, tanto si estos sistemas coinciden con los de las/os maestras/os que trabajan como tutoras/es de esas alumnas/os, como si se producen divergencias entre unos y otros.

\section{REFERENCIAS BIBLIOGRÁFICAS}

Abelson, R. (1979). Differences between belief systems and knowledge systems. Cognitive Science, $3(4), 355-366$.

Benítez, W. (2013). Concepciones sobre las matemáticas, su enseñanza y su aprendizaje de docentes en formación. Revista Científica, Edición Especial, 176-180. https://bit.ly/3n4IHNS

Blanco, L. (1996). Aprender a enseñar matemáticas: tipos de conocimiento. En J. Giménez, S. Llinares y V. Sánchez (eds.). El proceso de llegar a ser un profesor de primaria. Cuestiones desde la educación matemática (pp. 199-221). Comares.

Bramald, R., Hardman, F. \& Leat, D. (1995). Initial teacher trainees and their views of teaching and learning. Teaching \& Teacher Education, 11(1), 23-31.

Caballero, A., Blanco, L. J. y Guerrero, E. (2007). Las actitudes y emociones ante las matemáticas de los estudiantes para maestros de la facultad de educación de la universidad de Extremadura. En M. Camacho, P. Bolea, P. Flores, B. Gómez, J. Murillo y M. T. González (eds.). Investigación en Educación Matemática. Comunicaciones de los Grupos de Investigación. XI Simposio de la SEIEM (pp. 41-52). SEIEM. https://bit.ly/30gZkMP

Carrillo, J. (1998). Modos de resolver problemas y concepciones sobre la matemática y su enseñanza: metodología de la investigación y relaciones. Universidad de Huelva.

Civil, M. (1996). Pensando sobre las matemáticas y su enseñanza: Una experiencia con estudiantes para profesores de primaria. En J. Giménez, S. Llinares y V. Sánchez (eds.). El proceso de llegar a ser un profesor de primaria. Cuestiones desde la educación matemática (pp. 173-197). Comares.

Cristerna, C. (2019). Las creencias de los profesores sobre la enseñanza de la historia y su relación con la construcción de nociones espaciotemporales. Qurriculum, 32, 135-153. https://bit. ly/2NBwqzH

de la Fuente, G. y Sánchez, M. (2000). La socialización anticipada en la universidad. El caso de los estudiantes de magisterio y educación social. Revista de Educación, 321, 269-290. https://bit. ly/30gjCFW

de Vries, S., Van de Grift, W. y Jansen, E (2014). How teachers' beliefs about learning and teaching relate to their continuing professional development. Teachers and Teaching, 20(3), 338-357. https://bit.ly/2U4wijc

Escudero, I.; García, M.; Llinares, S. y Sánchez, V. (1993). Creencias epistemológicas sobre las matemáticas en los estudiantes para profesores de primaria. Enseñanza de las Ciencias, 11 (número extra 1), 317-318.

Feiman-Nemser, S. y Buchman, M. (1988). Lagunas en las prácticas de enseñanza de los programas de formación del profesorado. En L. Villar (dir.). Conocimiento, creencias y teorías de los profesores. Implicaciones para el currículum y la formación del profesorado (pp. 301-314). Marfil.

Feliu, M.; Vallés, A. y Cardona, G. (2016). Innovación en la formación inicial del profesorado: exploración de creencias sobre la enseñanza de las Ciencias Sociales en alumnos de Magisterio. Revista Electrónica Interuniversitaria de Formación del Profesorado, 19(3), 1-9. https://bit. $1 \mathrm{y} / 36 \mathrm{c} 8 \mathrm{Btg}$

Flores, P. (1998). Concepciones y creencias de futuros profesores sobre las matemáticas, su enseñanza y aprendizaje. Investigación durante las prácticas de enseñanza. Comares.

Foss, D. y Kleinsasser, R. (1996). Preservice elementary teachers' views of pedagogical and mathematical content knowledge. Teaching and Teacher Education, 12(4), 429-442. 
Freire, P. (2005). Pedagogía del oprimido. México: Siglo XXI.

Furinghetti, F. (1994). Practice what you preach: influencing preservice teachers' beliefs about mathematics. En L. Bazzini (ed.). Theory and Practice in Mathematics Education. Proceedings of the Fifth International Conference on Systematic Cooperation Between Theory and Practice in Mathematics Education (pp. 81-91). Grado.

González, M. y Fuentes, E. (1994). Las prácticas escolares en la formación del profesorado. Análisis y propuestas. Diputación Provincial de Lugo.

Green, T. (1971). The activities of teaching. McGraw Hill.

Jackson, P. (1992). La vida en las aulas. Morata.

Leinhardt, G. (1990). Capturing craft knowledge in teaching. Educational Researcher, 19(2), 18-25.

Llinares, S., Sánchez, V., García, M. y Escudero, I. (1995). Creencias y aprender a enseñar matemáticas. Universidad de Sevilla.

Marcelo, C. y Parrilla, A. (1991). El estudio de caso: Una estrategia para la formación del profesorado y la investigación didáctica. En C. Marcelo y otros (eds.). El estudio de caso en la formación del profesorado y la investigación didáctica (pp. 9-71). Universidad de Sevilla.

Merton, R. (1980). Teoría y estructura sociales. Fondo de Cultura Económica.

Pajares, M. (1992). Teachers' beliefs and educational research: cleaning up a messy construct. Review of Educational Research, 62(3), 307-332.

Palos, M., Ávalos, M., Flores, F. \& Montes, R. (2017). Creencias de madres y docentes sobre el aprendizaje de la lectoescritura en Educación Preescolar. Revista Actualidades Investigativas en Educación, 17(3), 1-21. https://bit.ly/3jhWdf4

Pérez, A. (1992). La función y formación del profesor/a en la enseñanza para la comprensión. Diferentes perspectivas. En J. Gimeno y A. Pérez (eds.). Comprender y transformar la enseñanza (pp. 398-429). Morata.

Porlán, R. (1989). Teoría del conocimiento, teoría de la enseñanza y desarrollo profesional. Las concepciones epistemológicas de los profesores. [Tesis doctoral, Universidad de Sevilla].

Rodríguez, C. (2002a). De alumna a maestra: Un estudio sobre socialización docente y prácticas de enseñanza. Universidad de Granada.

. (2002b). La socialización docente en las prácticas de enseñanza. Estudio de un caso. Revista Interuniversitaria de Formación del Profesorado, (45) 151-165. https://bit.ly/33aVbMb

Sánchez, V. y Llinares, S. (1996). Prácticas escolares habituales y situaciones de resolución de problemas: El caso de Carlota. En J. Giménez, S. Llinares y V. Sánchez (eds.). El proceso de llegar a ser un profesor de primaria. Cuestiones desde la educación matemática (pp. 223-248). Comares.

(1988). Un estudio de las creencias del futuro maestro en relación con las matemáticas: Influencia de las prácticas. En C. Marcelo (ed.). Avances en el estudio del pensamiento del profesor (pp. 207-216). Universidad de Sevilla.

Schutz, A. (1974). El problema de la realidad social. Amorrortu.

Shulman, L. (1989). Paradigmas y programas de investigación en el estudio de la enseñanza. Una perspectiva contemporánea. En M. Wittrock (ed.). La investigación de la enseñanza (pp. 9-91). Paidós.

Schoenfeld, A. (1991). On mathematics as sense-making: An informal attack on the unfortunate divorce of formal and informal mathematics. En J. Voss, D. Perkins \& J. Segal (eds.). Informal reasoning and instruction (pp. 311-343). Lawrence Erlbaum.

Struyven, K., Dochy, F. y Janssens, S. (2010). 'Teach as you preach': the effects of student-centred versus lecture-based teaching on student teachers' approaches to teaching. European Journal of Teacher Education, 33(1), 43-64. https://bit.ly/3iHYZNc

Swinson, K. \& Shield, M. (1994). Practice what you preach: influencing preservice teachers' beliefs about mathematics. En J. Ponte y J. Matos (eds.). Proceedings of the eighteenth International Conference for PME (pp. 321-328). Universidad de Lisboa. 
Thompson, A. (1992). Teacher's beliefs and conceptions: a synthesis of the research. En D. Grouws (ed.). Handbook on mathematics teaching and learning (pp. 127-146). MacMillan.

Vásquez, A. y Martínez, I. (1996). La socialización en la escuela. Una perspectiva etnográfica. Paidós.

Vila, A. y Callejo, M. (2005). Matemáticas para aprender a pensar. El papel de las creencias en la resolución de problemas. Narcea.

Wilcox, S., Lanier, P., Schram P. \& Lappan, G. (1992). Influencing beginning teachers' practice in mathematics education: Confronting constraints of knowledge, beliefs and context. Michigan State University.

Wilcox, S., Schram P., Lappan, G. y Lanier, P. (1991). The role of a learning community in changing preservice teacher's knowledge and beliefs about mathematics education. For the Learning of Mathematics, 11(3), 31-39. https://bit.ly/3zqT1WQ 
